\title{
LYMPHOCYTE-RICH PLEURAL LIPOSARCOMA MIMICKING PERICARDIAL CYST
}

Mobeen Iqbal, MD, ${ }^{\mathrm{a}}$ Jeffrey Posen, MB, BCh, ${ }^{\mathrm{b}}$ Tawfiqul Alam Bhuiya, MD, ${ }^{\mathrm{b}}$ Rudy P. Lackner, MD, ${ }^{\mathrm{c}}$ Harry N. Steinberg, MD, and Leonard J. Rossoff, MD, ${ }^{\mathrm{a}}$ New Hyde Park, $N Y$

Liposarcomas are the second most common histologic type of soft tissue sarcomas. ${ }^{1}$ Common sites of involvement include the lower extremities and retroperitoneum. Primary intrathoracic liposarcomas are rare with a preference for the mediastinum. ${ }^{1}$ Very few cases of primary involvement of lung and pericardium have been reported, including only 9 cases of pleural liposarcomas. ${ }^{2}$ We review a unique case of lympho-

From the Division of Pulmonary and Critical Care Medicine, ${ }^{\mathrm{a}}$ Department of Pathology, and Division of Cardiothoracic Surgery, ${ }^{\mathrm{c}}$ Long Island Jewish Medical Center, New Hyde Park, NY.

Received for publication March 1, 2000; accepted for publication March 6, 2000.

Address for reprints: Leonard J. Rossoff, MD, Division of Pulmonary and Critical Care Medicine, Long Island Jewish Medical Center, The Long Island Campus of the Albert Einstein College of Medicine, Room C-20, 270-05 76th Ave, New Hyde Park, NY 11042.

J Thorac Cardiovasc Surg 2000;120:610-2

Copyright $\odot 2000$ by The American Association for Thoracic Surgery

$0022-5223 / 2000 \$ 12.00+0 \quad \mathbf{1 2 / 5 4 / 1 0 6 9 7 1}$

doi: $10.1067 / \mathrm{mtc} .2000 .106971$ cyte-rich pleural liposarcoma, which mimicked a pericardial cyst on computed tomographic (CT) scan. Histopathologic examination revealed a recently described lymphocytic-rich variant, which might be mistaken for lymphoma. ${ }^{3}$

Clinical summary. A 78-year-old white man with a 40 pack-year history of smoking was seen for an asymptomatic opacity detected on a routine chest x-ray film. A chest radiograph 4 months earlier had shown no abnormalities. His medical history was significant for controlled atrial fibrillation, adult-onset diabetes mellitus, hypertension, and benign prostatic enlargement. He specifically denied cough, chest pain, and all constitutional symptoms. He reported dyspnea on moderate exertion, which had been stable for many months. His medications included lisinopril, metformin, digoxin, doxazosin mesylate, aspirin, and felodipine.

On physical examination, vital signs were within normal limits and no adenopathy or jugular venous distention was detected. Examination of the chest was unremarkable, as was the rest of the physical examination. The chest radiograph revealed an opacity abutting the lower right border of the heart (Fig 1, A). A CT scan of the chest revealed a cystic opacity in the major fissure, apparently attached to the pericardium. The location and CT appearance were suggestive of a pericardial 

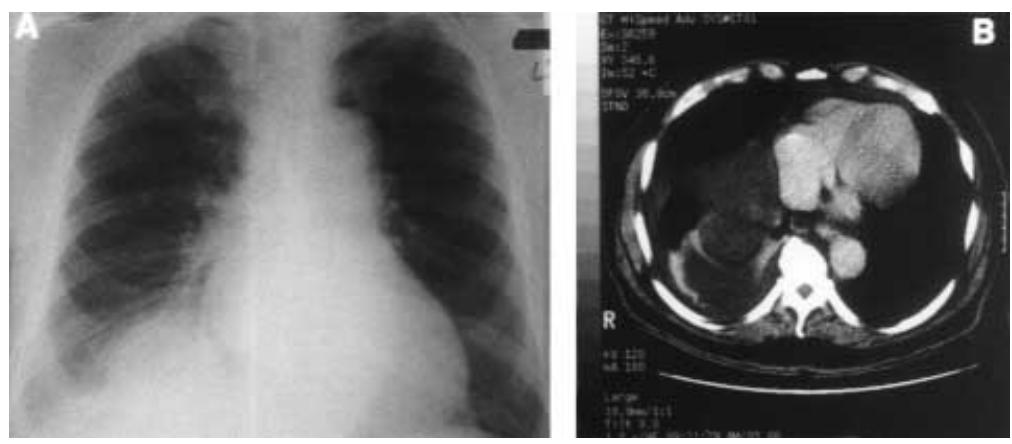

Fig 1. A, Chest x-ray film showing a well-circumscribed opacity in the right cardiophrenic angle. B, CT scan revealing the cystic opacity of fluid density in the right hemithorax abutting the pericardium with moderate-sized pleural effusion.

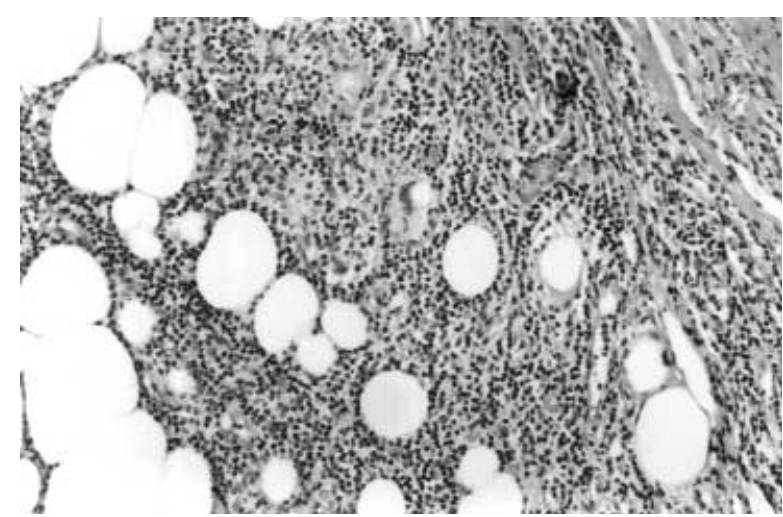

Fig 2. An area of dense lymphocytic infiltration obscuring underlying neoplasm.

cyst. A 1-cm nodule was also detected in the right lower lobe. No intervention was advised aside from another radiographic evaluation in 3 to 4 months.

Two months later he had persistent pleuritic chest pain on the right side without fever or cough. A CT scan at that time revealed a new moderate-sized right-sided pleural effusion and an increase in the size of the cyst (Fig 1,B). A thoracentesis revealed an exudate with no malignant cells detected. The patient underwent surgical resection of the cyst. Intraoperatively, the lesion was found to be a dumbbellshaped rubbery mass arising from the medial aspect of the diaphragmatic pleura extending to the inferior pericardium. The mass, along with part of the diaphragm and pericardium, was resected. The diaphragm was plicated and a wedge resection of the right lower lobe nodule was performed.

Gross examination revealed a soft rubbery encapsulated mass measuring $11 \times 8 \times 6.5 \mathrm{~cm}$. Histologic sections revealed lymphocyte-rich areas partially obscuring the neoplastic background, which varied from area to area (Fig 2). The background comprised loose connective tissue interspersed with more dense areas of lymphoid proliferation and fibrovascular connective tissue with spindle cells and abundant myxoid material. There were foci of more typical look-

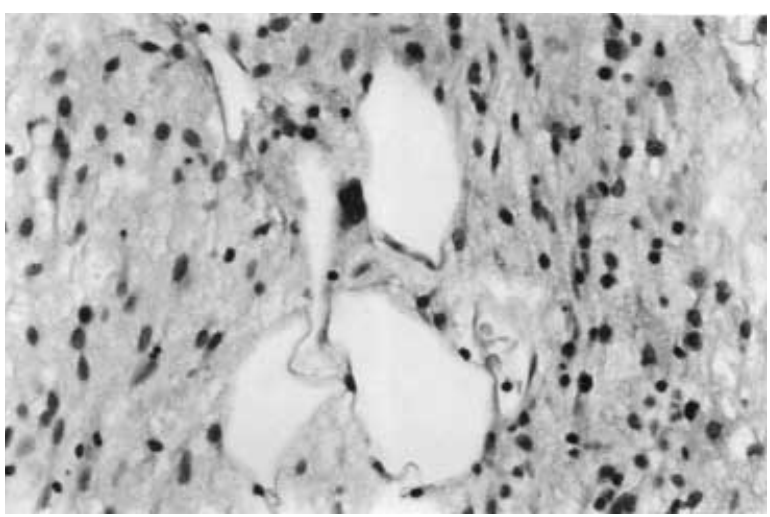

Fig 3. Lipoblasts in myxoid stroma and more mature looking fatty tissue.

ing liposarcoma and scattered atypical cells resembling lipoblasts with hyperchromatic nuclei and vacuolated cytoplasm (Fig 3). The tumor was adherent to the lung pericardium and diaphragm without any microscopic invasion of these structures. The small nodule in the right lower lobe was a well-differentiated carcinoid, with orderly architecture and acinar formation.

The patient had an uneventful postoperative course and subsequently underwent external beam radiation to the region of involvement.

Discussion. Liposarcomas are usually well circumscribed and lobulated. Despite the name, they generally do not originate from adipose tissue. The cell of origin is a primitive mesenchymal cell, which has the property of lipogenesis. ${ }^{2}$ Four histologic subtypes have been described: well differentiated, myxoid (most common), pleomorphic, and dedifferentiated. ${ }^{3}$ Pleural liposarcomas are rare, and the previously described 9 cases have no characteristic presentation other than a possible male predilection. The lymphocyte-rich variant of liposarcoma is a recently described entity with most of the tumors originating retroperitoneally or in other locations. ${ }^{3,4} \mathrm{We}$ believe ours is the first case report of intrathoracic lymphocyte-rich liposarcoma. 
Surgery and radiotherapy have been the mainstays of treatment. Resection, even with wide margins, has a reported frequency of local recurrence of $70 \%$ to $90 \% .^{2}$ In one study, the 5 -year survival of patients treated with surgery alone was $25 \%$, and in unresectable tumors survivals as high as $45 \%$ has been reported with radiation alone. ${ }^{5}$ Chemotherapy generally is not recommended in the treatment of liposarcomas because its role is unclear. Histology also appears to affect survival. Well-differentiated and myxoid subtypes have a local control rate exceeding $90 \%$ at 10 years with combined surgery and radiotherapy and less tendency to metastasize. ${ }^{6}$ Pleomorphic liposarcoma is a highly malignant tumor with a high local recurrence rate of $37 \%$ and a metastasis rate of $41 \%$ at 10 years. These biologic factors also reflect in progressively decreasing 10 -year survivals from $87 \%$ for well-differentiated lesions, through $76 \%$ for myxoid tumors, to $39 \%$ for pleomorphic tumors. ${ }^{6}$

In conclusion, pleural liposarcomas are rare tumors. Clinical presentation is variable and in our case initially mimicked a pericardial cyst. The importance of recognizing a category of lymphocyte-rich liposarcoma is not to confuse the diagnosis with that of a benign or malignant lymphoproliferative disorder. Surgical excision with adjuvant radiation is the currently recommended treatment.

\section{REFERENCES}

1. Enzinger FM, Weiss SW. Soft tissue tumors. 2nd ed. St Louis: Mosby; 1988. p. 346-82.

2. Wong WW, Pluth JR, Grado GL, Schild SE, Sanderson DR. Liposarcoma of the pleura. Mayo Clin Proc 1994;69:882-5.

3. Argani P, Facchetti F, Inghirami G, Rosai J. Lymphocyte-rich well-differentiated liposarcoma: report of nine cases. Am J Surg Pathol 1997;21:884-95.

4. Krauss M, Gillou L, Fletcher CD. Well-differentiated inflammatory liposarcoma: an uncommon and easily overlooked variant of a common sarcoma. Am J Surg Pathol 1970;20:2-9.

5. Brasfield RD, Das Gupta TK. Liposarcoma. CA Cancer J Clin 1970;20:2-9.

6. Zagars GK, Goswitz MS, Pollack A. Liposarcoma: outcome and prognostic factors following conservation surgery and radiation therapy. Int J Radiat Oncol Biol Phys 1996;36:311-9. 